\title{
Interplay between electrical transport properties of GeMn thin films and Ge substrates
}

\author{
N. Sircar, ${ }^{1}$ S. Ahlers, ${ }^{1}$ C. Majer, ${ }^{1}$ G. Abstreiter, ${ }^{1}$ and D. Bougeard ${ }^{1,2}$ \\ ${ }^{1}$ Walter Schottky Institut, Technische Universität München, Am Coulombwall 4, D-85748 Garching, Germany \\ ${ }^{2}$ Institut für Experimentelle und Angewandte Physik, Universität Regensburg, D-93040 Regensburg, Germany \\ (Received 5 November 2010; revised manuscript received 7 February 2011; published 18 March 2011)
}

\begin{abstract}
We present evidence that electrical transport studies of epitaxial $p$-type GeMn thin films fabricated on highresistivity Ge substrates are severely influenced by parallel conduction through the substrate, related to the large intrinsic conductivity of Ge due to its small band gap. Anomalous Hall measurements and large magnetoresistance effects are completely understood by taking a dominating substrate contribution as well as the measurement geometry into account. It is shown that substrate conduction persists also for well-conducting, degenerate, $p$-type thin films, giving rise to an effective two-layer conduction scheme. Using $n$-type Ge substrates, parallel conduction through the substrate can be reduced for the $p$-type epilayers, as a consequence of the emerging $p n$-interface junction. GeMn thin films fabricated on these substrates exhibit a negligible magnetoresistance effect. Our study underlines the importance of a thorough characterization and understanding of possible substrate contributions for electrical transport studies of GeMn thin films.
\end{abstract}

DOI: 10.1103/PhysRevB.83.125306

PACS number(s): 73.50.-h, 75.50.Pp, 75.47.-m, 73.61.-r

\section{INTRODUCTION}

In recent years the emerging field of spintronics has led to the search for novel materials exhibiting ferromagnetic and semiconducting properties at the same time, since such ferromagnetic semiconductors would allow for the integration of new application schemes into established semiconductor technologies. GeMn seems to be a very promising candidate in the class of ferromagnetic semiconductors for its compatibility with the mainstream silicon technology. Recent works consistently demonstrated the possibility of preparing GeMn thin films by molecular beam epitaxy (MBE) without secondary phase separation, but with a strong tendency toward the formation of Mn-rich nanometer-sized clusters. ${ }^{1-4}$ These clusters exhibit a Curie temperature about or even above room temperature (RT), which would be important for device applications. In an earlier work it was claimed that magnetic properties may be controlled through electric gating, suggesting that charge carriers mediate the magnetic exchange interactions. ${ }^{5}$ Therefore the electrical properties in an external magnetic field, i.e., the magnetoresistance (MR) and the anomalous Hall effect (AHE) are considered an important fingerprint of a magnetic semiconductor. However, in the same degree as there is a consistent picture of the nanostructure of GeMn thin films, their fingerprint in magnetotransport measurements still lacks such a coherent description in literature. For example, MR effects reaching from several thousand percent being positive ${ }^{2}$ to a few percent being negative $e^{4,6,7}$ have been reported. Similarly, Hall-effect measurements sometimes yield a large contribution of the AHE on the one hand, ${ }^{6,8}$ but also a diminishing contribution washed out by the ordinary Hall effect on the other. ${ }^{9}$ In many cases the interpretation of these results interestingly does not correlate with the sample magnetization, particularly regarding its saturation and hysteresis effects, which are often absent in transport measurements. Recently it was pointed out by Zhou et al. ${ }^{9}$ that part of these reports may be understood in a regime of parallel conduction of two charge-carrier types, owing to the role of $\mathrm{Mn}$ as a deep two-level acceptor in Ge, without being related to the magnetic nature of GeMn thin films in itself.
In this study, we would like to highlight the possibility that the peculiar transport properties observed in some GeMn transport studies might not only be caused by the alloying of Ge with Mn, but by parallel conduction through the employed substrates. While high-purity substrates of semiconductors such as $\mathrm{Si}$ or $\mathrm{GaAs}$ exhibit RT resistivities greater than $10^{3} \Omega \mathrm{cm}$, this is not the case for Ge substrates. Since intrinsic conduction in Ge already becomes important around RT due to its small band gap, RT resistivities are intrinsically limited around $50 \Omega \mathrm{cm} .{ }^{10}$ This upper limit is already reached with impurity concentrations as low as $10^{13} \mathrm{~cm}^{-3}$. ${ }^{11}$ Substrates with higher purity are commercially not available. We will show that the electrical properties of epitaxial GeMn thin films fabricated by solid-source MBE on such high-purity $\mathrm{Ge}$ substrates can severely be influenced by parallel conduction through the substrate. To furthermore demonstrate the effects of parallel conduction, we studied a system of non-magnetic, degenerately doped Ge:B epitaxial layers grown on these highpurity Ge substrates. Some of these results have a remarkable resemblance to previously published data on magnetic GeMn thin films, ${ }^{9,12}$ although our Ge:B films do not show any sign of magnetism other than common diamagnetism. We will give a two-layer model accounting for the parallel conduction through the substrate, which sufficiently well describes the experimental magnetotransport results in those types of thin films.

\section{EXPERIMENTAL}

The investigated samples were fabricated with solid-source MBE under ultrahigh vacuum conditions at a base pressure of $5 \times 10^{-11}$ mbar. We used high-resistivity $\mathrm{Ge}(001)$ substrates with a RT resistivity larger than $40 \Omega \mathrm{cm}$ and a thickness of approximately $500 \mu \mathrm{m}$. These substrates are specified to exhibit $n$-type conduction, due to antimony impurities dissolved into the Ge crystal during its fabrication. However, we would like to note that the vendors specification of this $n$-type conduction is only true about RT. In fact, the substrate undergoes a transition to $p$-type conduction below 
(a)

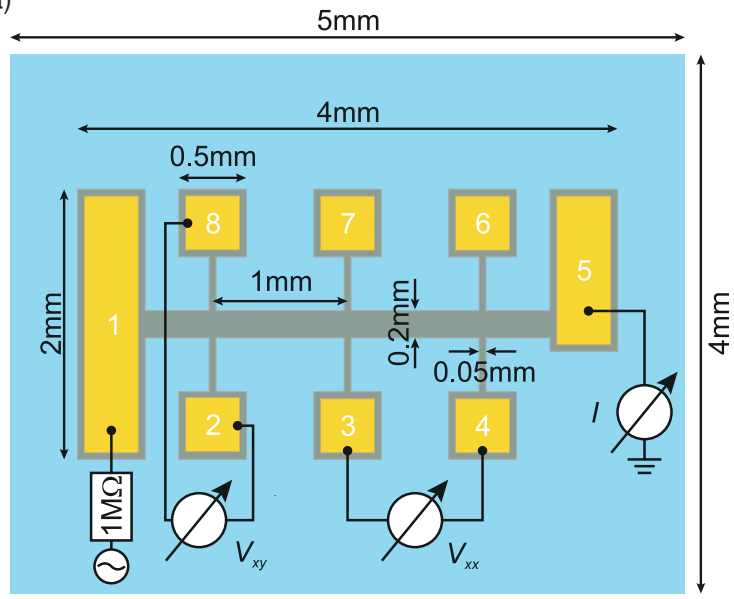

(b)

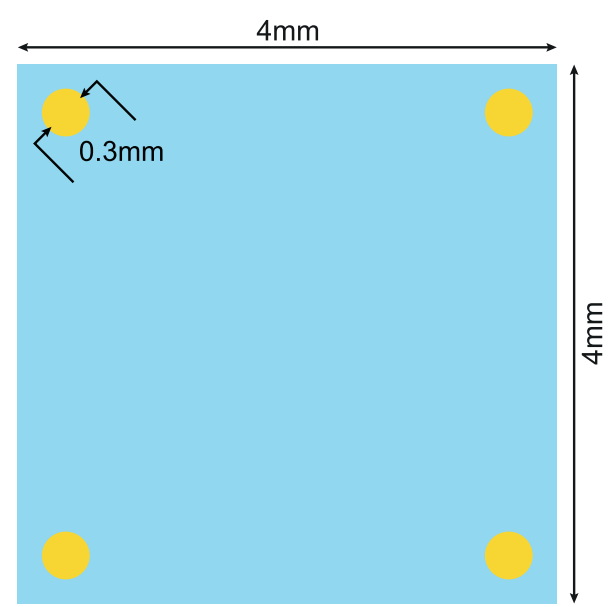

FIG. 1. (Color online) (a) Scaled schematic of samples with Hall bar mesa. The principle setup for measuring the longitudinal ( $\left.V_{x x}\right)$ and Hall $\left(V_{x y}\right)$ voltage as well as total current $(I)$ is also indicated. (b) Geometry of samples where the van der Pauw method has been employed.

RT. This indicates the presence of a majority of residual acceptorlike impurities. Hence, the substrate may suffer from considerable autocompensation. At RT, the conduction behavior is dominated by intrinsic charge carriers, primarily by electrons due to their smaller effective mass, and is therefore $n$ type.

Prior to the growth of all thin films, a 80-nm-thick, undoped Ge buffer layer was deposited. The GeMn sample was grown by codeposition of $\mathrm{Mn}$ and $\mathrm{Ge}$ at a Ge growth rate of $r_{\mathrm{Ge}}=0.08 \AA_{\mathrm{s} \mathrm{s}}^{-1}$ and at a constant substrate temperature $T_{\mathrm{S}}=60^{\circ} \mathrm{C}$ to avoid the formation of intermetallic secondary phases. The film thickness amounts to $200 \mathrm{~nm}$ with a total Mn concentration of 5\%. A thorough characterization of the structural and magnetic properties of this sample may be found elsewhere. ${ }^{1}$

For comparison, we also fabricated a nonmagnetic $p$-type $\mathrm{Ge}$ thin film using a boron effusion cell. This sample was fabricated at $r_{\mathrm{Ge}}=0.3 \AA^{\circ} \mathrm{s}^{-1}$ and $T_{\mathrm{S}}=360^{\circ} \mathrm{C}$ with a thickness of $200 \mathrm{~nm}$. The B concentration of $5 \times 10^{19} \mathrm{~cm}^{-3}$ was chosen to be well above the insulatorto-metal transition. ${ }^{13}$

For transport measurements an approximately 450-nmdeep Hall bar (HB) mesa was defined by standard lithography methods and wet-chemical etching. Geometrical details are given in Fig. 1(a). The longitudinal and Hall resistances, $R$ and $R_{x y}$, were determined by applying a current $I$ along the $\mathrm{HB}$ and measuring the longitudinal and Hall voltages, $V_{x x}$ and $V_{x y}$, in a standard, quasi-dc lock-in setup using an additional $1 \mathrm{~T} \Omega$ input impedance voltage amplifier before the lock-in. As will be introduced in Sec. IIIC, some samples were also investigated via the van der Pauw method in the geometry shown in Fig. 1(b).

Temperature-dependent resistance measurements without applied magnetic fields were performed with a heatable sample stick inserted in a liquid-helium dewar. Field-dependent measurements were performed in a variable-temperature magnet cryostat, with the magnetic field applied perpendicular to the sample surface.

\section{RESULTS AND DISCUSSION}

\section{A. Nondegenerate GeMn on high-resistivity Ge substrates}

Figure 2 depicts the sample resistance of the bare highresistivity Ge substrate sample as a function of temperature. We can identify the three distinct regions well known for nondegenerate semiconductors, i.e., the freeze-out of extrinsic charge carriers, the extrinsic, and the onset of the intrinsic range.

Also shown in Fig. 2 is the resistance measurement of the GeMn thin film grown on the high-resistivity Ge substrate. When comparing the two samples, we notice that the resistance of both samples is of the same order of magnitude and has a very similar temperature dependence. This becomes more evident in an Arrhenius plot of the resistance depicted in the inset of Fig. 2. Both curves exhibit the same linear slope

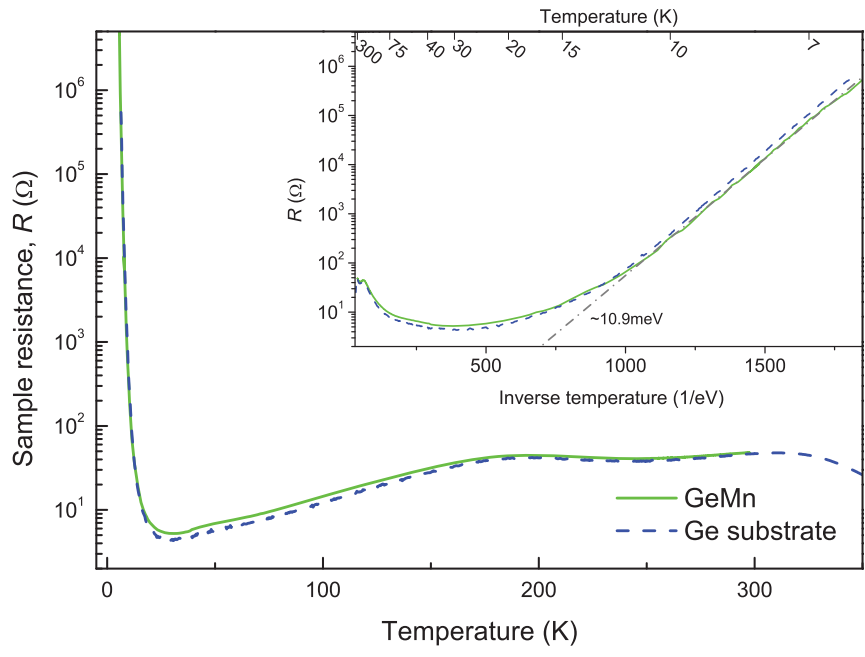

FIG. 2. (Color online) Sample resistance versus temperature for the GeMn sample (green solid) and the Ge substrate reference (blue dashed). Inset: Same data as function of inverse temperature. A straight line (grey dash-dotted) corresponding to an activation energy of $10.9 \mathrm{meV}$ can be fitted to the extrinsic freeze-out. 
in the extrinsic freeze-out regime, which corresponds to a thermal activation energy of $E_{\mathrm{A}}=10.9 \mathrm{meV}$ for the dopant impurities. ${ }^{14}$ This is in good agreement with the activation energy of shallow impurities in Ge. It does not correspond with the activation energy of $\mathrm{Mn}$ in $\mathrm{Ge}$, which is expected to be a two-level deep band-gap acceptor with $E_{\mathrm{A}}=160$ and $370 \mathrm{meV}$, respectively. ${ }^{15}$ It rather seems that in both samples the residual impurities dissolved in the Ge substrate dominate the measurements.

Figure 3(a) shows the MR effect of the GeMn sample versus external magnetic field $B$ for various temperatures, calculated according to the convention

$$
\operatorname{MR}[\%]=\frac{R(B)-R(0)}{R(0)} \times 100 .
$$

The MR effect is positive and exhibits a paraboliclike dependence for weak fields tending toward a linear dependence at higher fields, without any signs of saturation. For higher temperatures the MR effect gradually decreases in its magnitude. Similar results on the MR effect in GeMn have already been reported. $^{2}$

The orbital MR of the semiclassical Boltzmann transport theory cannot be responsible for the positive MR effect

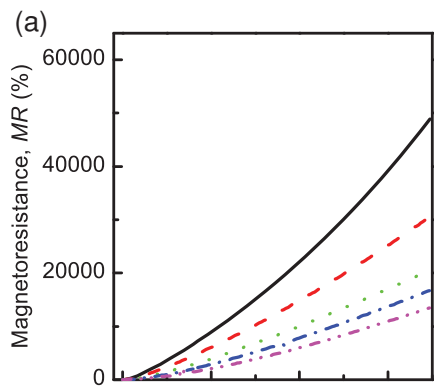

(b)
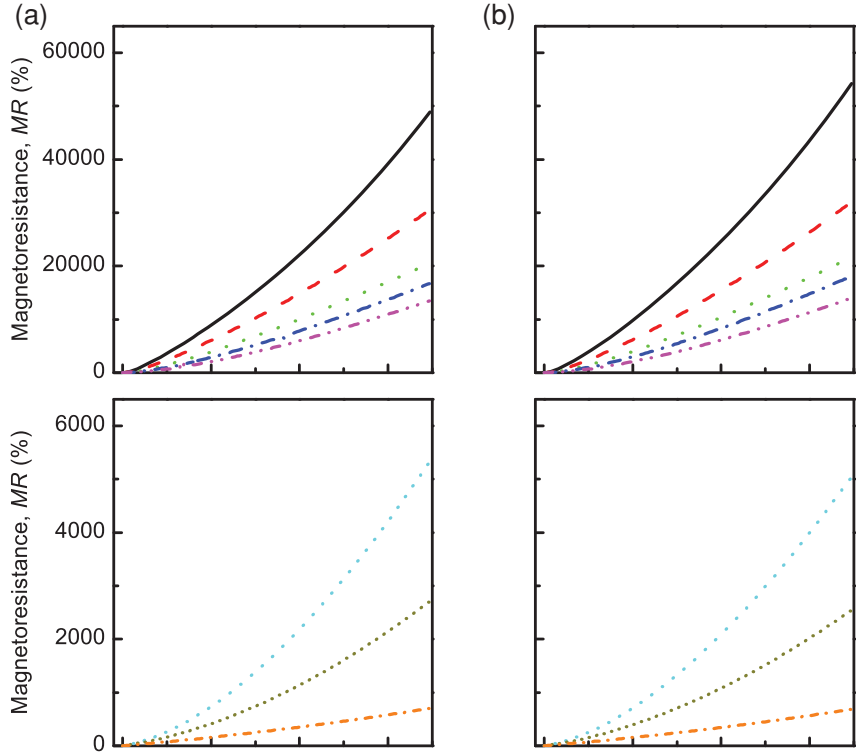

(c)

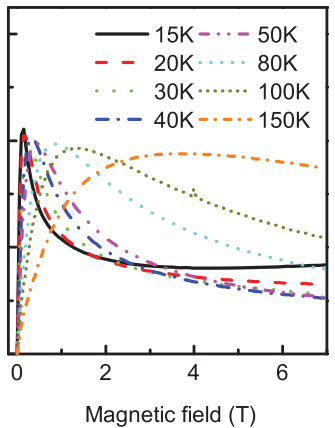

FIG. 3. (Color online) (a) MR for various temperatures for the GeMn sample. (b) MR for the bare high resistivity Ge substrate. (c) Tangent of the Hall angle for (left) the GeMn sample and (right) the Ge substrate. The color code is the same for all panels. depicted in Fig. 3(a), since the order of magnitude of the MR is too large. In fact, an orbital MR effect would also not explain the nonsaturating character of the observed MR at large fields. ${ }^{16}$ A connection of the MR to the magnetic nature of the GeMn epilayer can be ruled out for the same reason, as its magnetization saturates at fields about $2 \mathrm{~T}^{1}{ }^{\mathrm{J}} \mathrm{Jamet}$ et $a .^{2}$ proposed the occurrence of a geometrically enhanced MR effect $^{17}$ to account for the large magnitude and the linear increase at high fields of the MR in their GeMn sample, stemming from the presence of highly conducting Mn-rich inclusions. Such an inhomogeneous semiconductor can indeed exhibit extremely large, nonsaturating MR. ${ }^{18-20}$ However, we obtain essentially equal results for the magnitude as well as field and temperature dependence of the MR of the bare Ge substrate, as shown in Fig. 3(b).

The left panel in Fig. 3(c) depicts the tangent of the Hall angle of the GeMn sample, defined as $\rho_{x y} / \rho_{x x}$, with $\rho_{x y}$ and $\rho_{x x}$ being the Hall and longitudinal resistivities, respectively. The Hall angle gives a more direct estimate of possible magnetization-induced contributions to the ordinary Hall effect than the common Hall curve. The Hall angle increases steeply with field, tending toward a saturation at higher fields. Similar results were found by other groups for the GeMn material system and were either attributed to a magnetization-induced AHE (Ref. 2) or related to the multiple Mn acceptor energy states leading to an effective two-band-like conduction. ${ }^{9}$ Our undoped, nonmagnetic Ge substrate exhibits the same Hall angle behavior as can be seen in the right panel of Fig. 3(c).

The data presented on the GeMn thin film in Figs. 2 and 3 show a strong similarity to the underlying substrate. This suggests that neither the inhomogeneity, magnetic nature, nor the presence of Mn acceptors in the GeMn thin film leads to the observed transport properties in the present case. We conclude that the transport properties of our GeMn sample do emerge from parallel conduction through the substrate.

This dominating contribution of the substrate can be understood, when one considers the system of an epitaxially fabricated GeMn thin film on top of the high-resistivity Ge substrate as two parallel conducting resistors. For an independent determination of the transport properties of the epilayer without contributions from the substrate layer, the resistance of the GeMn epilayer has to be at least a factor of 10 smaller than that of the substrate. A comparison of the thicknesses of these two layers implicates that the epilayer resistivity then has to be smaller by a factor of $10^{4}$ than the resistivity of the Ge substrate. Considering the RT value of the substrate resistivity of about $40 \Omega \mathrm{cm}$, this in turn means that the GeMn epilayer resistivity has to be in the $10^{-3} \Omega \mathrm{cm}$ regime. For the present GeMn epitaxial layer, having a hole density around $10^{19} \mathrm{~cm}^{-3}$ (cf. Sec. III D), but nevertheless being nondegenerate, that would demand RT mobilities on the order of a few $10^{2} \mathrm{~cm}^{2} \mathrm{~V}^{-1} \mathrm{~s}^{-1}$. However, since GeMn thin films exhibit a very inhomogeneous nanostructure, ${ }^{1-4}$ such a high mobility cannot be expected. In fact, mobilities of that order of magnitude are only reached in conventional $p$-type doped Ge with similar hole concentrations, when the dopants are homogeneously diluted in the host matrix. ${ }^{21}$ In essence, because of the low conductivity of nondegenerate GeMn thin films, one cannot determine the transport properties 
of the GeMn epilayer in a straightforward manner, when it is fabricated on high-resistivity Ge substrates.

\section{B. Degenerate epitaxial $p$-type Ge on high-resistivity Ge substrates}

We now would like to address whether an electrical transport characterization of degenerately doped, $p$-type GeMn thin films on high resistivity Ge substrates, i.e., thin films with carrier concentrations clearly above $10^{19} \mathrm{~cm}^{-3}$, is feasible. In order to separate phenomena related to the magnetization or nanostructure from those related to parasitic conduction through the substrate, we explored degenerately doped, nonmagnetic Ge:B epilayers as a model system. Since B opposed to $\mathrm{Mn}$ is not a deep, but a shallow acceptor in $\mathrm{Ge}$, a doping concentration of $5 \times 10^{19} \mathrm{~cm}^{-3}$ lies well above the Mott insulator-to-metal transition, and is therefore sufficiently large to deliver thin films with degenerate, metalliclike conduction properties. Figure 4 shows the temperature dependence of the resistance of the $\mathrm{Ge}: \mathrm{B}$ sample. Interestingly, only at temperatures below approximately $10 \mathrm{~K}$ do the measurements reflect the metallic character of the epilayer, as the resistance enters a constant value regime. Above this temperature the curve quickly traces the measurement of the Ge substrate, which is also depicted for a comparison.

The magnetic field dependence of the longitudinal resistance of the Ge:B sample is depicted in Fig. 5(a) for different temperatures. At temperatures below $9 \mathrm{~K}$, the resistance shows little field dependence, yielding a MR effect which does not exceed $3 \%$ at $4.2 \mathrm{~K}$ and $7 \mathrm{~T}$. At approximately $9 \mathrm{~K}$ we observe the onset of the decrease of the zero-field resistance with increasing temperature, as already depicted in Fig. 4. However, the resistance now rises quickly with increasing magnetic field, hence giving an increased MR effect. Eventually, at high fields the resistance tends to saturate at the $4.2 \mathrm{~K}$ value. With increasing measurement temperature this saturation is shifted toward higher fields, while at the highest temperatures full

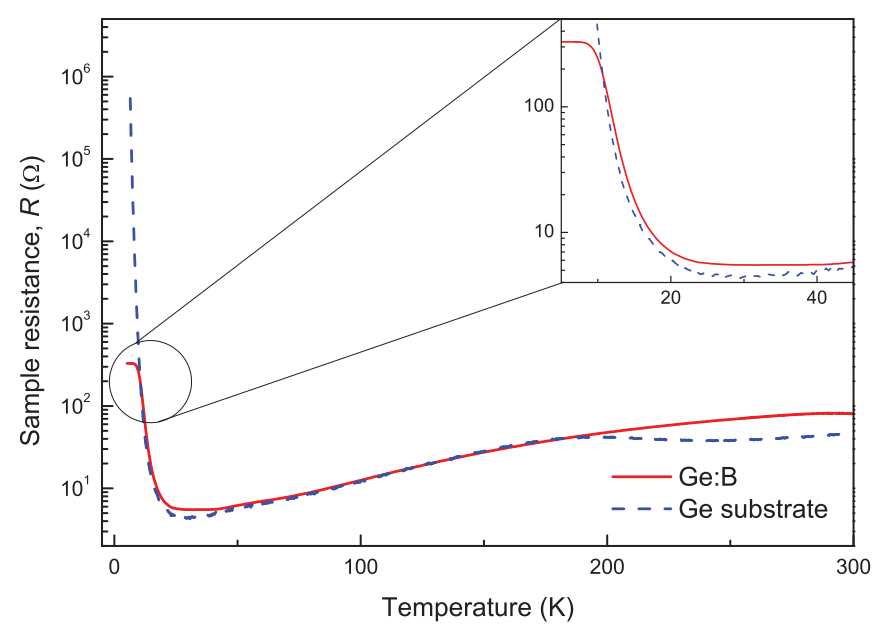

FIG. 4. (Color online) Sample resistance as a function of temperature for the Ge:B sample (red solid) with a doping concentration of $5 \times 10^{19} \mathrm{~cm}^{-3}$. The reference measurement of the substrate (blue dashed) is also depicted. The inset shows a close-up for small temperatures, indicating the metallic conductance of the Ge:B sample, when parallel conduction through the substrate ceases.
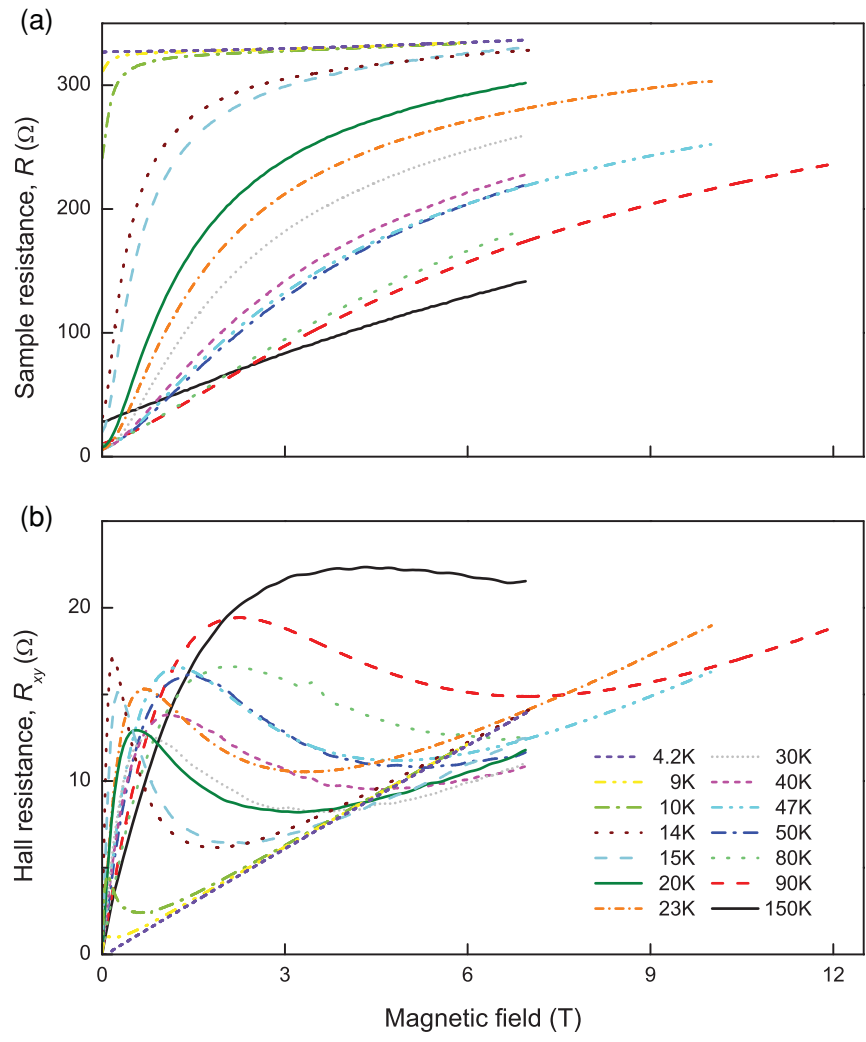

FIG. 5. (Color online) (a) Sample resistance versus magnetic field of the Ge:B sample. (b) Hall resistance of the Ge:B sample. The dip of $R$ at zero field and the peak of $R_{x y}$ at low fields mark the onset of parallel conduction through the substrate at $9 \mathrm{~K}$. The color code is the same in both panels.

saturation is not reached anymore within the investigated field range.

The Hall effect of the Ge:B sample is shown in Fig. 5(b). Below $9 \mathrm{~K}$ we observe a linear Hall effect. At temperatures of $9 \mathrm{~K}$ and above, the field dependence drastically changes: For small field values we first observe a strong increase of the Hall slope. Upon increasing the field, the Hall effect shows a peaklike maximum and then approaches the Hall curve measured for $4.2 \mathrm{~K}$ asymptotically.

The transport behavior of the Ge:B film, depicted in Figs. 4 and 5 is not in line with the metallic character of the epilayer. We can rather identify two distinct temperature regimes with different properties below and above $9 \mathrm{~K}$. Similar results for the Hall effect and field dependence of longitudinal resistivity, which can be separated into two temperature regimes, were found for the GeMn material systems in degenerate thin films prepared by ion implantation. ${ }^{12}$ They were interpreted in terms of a two-band-like conduction scheme, accounting for possible electronic ground and excited states of $\mathrm{Mn}$ in $\mathrm{Ge} .{ }^{9}$ In contrast, our results in the two distinct regimes are naturally explained by assuming parallel conduction through the substrate: Below $9 \mathrm{~K}$ parallel conduction is not present, since the substrate resistance gets very large, whereas the resistance of the metallic epilayer does not change. Assuming now that conduction only takes place in the 200-nm-thick Ge:B epilayer, we can extract a hole density of $1.54 \times 10^{19} \mathrm{~cm}^{-3}$, in fair agreement with the nominal concentration value. Furthermore, the carrier mobility 
amounts to the relatively small value of $\mu=310 \mathrm{~cm}^{2} \mathrm{~V}^{-1} \mathrm{~s}^{-1}$. Due to the general proportionality between the orbital MR and carrier mobility, the small MR effect would therefore also be in line with conduction through the metallic $\mathrm{Ge}: \mathrm{B}$ epilayer. The transport measurements of the Ge:B sample can undoubtedly be attributed solely to the Ge:B epilayer in the temperature regime below $9 \mathrm{~K}$. For temperatures above $9 \mathrm{~K}$ we need to include substrate contributions for an interpretation of the magnetotransport data. At low fields, conduction will mostly take place in the substrate because of its smaller resistance. At large fields, conduction through the substrate will quickly cease, because its MR gets larger. Then most of the current flows through the epilayer. Thus, in the Hall as well as the MR measurement, we probe the substrate properties at a small magnetic field and the Ge:B epilayer properties at a high field, leading to the described peaking and saturation effects. The decreasing tendency of saturation at a high magnetic field with increasing measurement temperature comes from the weakening of MR of the Ge substrate. The Ge:B sample thus has to be regarded as a system of two conducting layers with different galvanomagnetic responses.

This phenomenological interpretation is supported by a description of the magnetotransport data with a two-layer conduction model. It is based on the assumption that each conducting layer can be described by its individual resistivity tensor, which reduces to a $2 \times 2$ matrix in the case where the magnetic field is normal to the plane of carrier motion. Since the two layers are not equally thick, in the following expressions we will give sheet resistivities rather than bulk resistivities to maintain generality. The resulting components of the sheet resistivity tensor of the combined two-layer system have the form

$$
\begin{gathered}
\rho_{x x}=\rho_{y y}=\frac{\rho_{1, x x}\left(\rho_{2, x x}^{2}+\rho_{2, x y}^{2}\right)+\rho_{2, x x}\left(\rho_{1, x x}^{2}+\rho_{1, x y}^{2}\right)}{\left(\rho_{1, x x}+\rho_{2, x x}\right)^{2}+\left(\rho_{1, x y}+\rho_{2, x y}\right)^{2}}, \\
\rho_{x y}=-\rho_{y x}=\frac{\rho_{1, x y}\left(\rho_{2, x x}^{2}+\rho_{2, x y}^{2}\right)+\rho_{2, x y}\left(\rho_{1, x x}^{2}+\rho_{1, x y}^{2}\right)}{\left(\rho_{1, x x}+\rho_{2, x x}\right)^{2}+\left(\rho_{1, x y}+\rho_{2, x y}\right)^{2}} .
\end{gathered}
$$

The quantities with subscripts 1 and 2 correspond to the tensor components of the Ge:B epilayer and the Ge substrate layer, respectively. We restrict ourselves to a semiempirical application of the above equations for a computation of the Hall and MR effects of the Ge:B sample for different temperatures. The parameters $\rho_{1 / 2, x x}$ and $\rho_{1 / 2, x y}$ entering this computation are taken from measurements: The contributions $\rho_{1, x x}$ and $\rho_{1, x y}$ of the Ge:B epilayer correspond to the $4.2 \mathrm{~K}$ measurement curves of the $\mathrm{Ge}: \mathrm{B}$ sample as were shown in Figs. 5(a) and 5(b). We assume they do not vary with increasing temperature, which is justified by the metallic character of this epilayer (see also Sec. IIID), therefore using them for all temperatures we investigate. The contributions of the $\mathrm{Ge}$ substrate, $\rho_{2, x x}$ and $\rho_{2, x y}$, are taken from the measurements depicted in Figs. 3(b) and 3(c) for each corresponding temperature.

Figure 6 shows the results of the computation of $\rho_{x x}$ and $\rho_{x y}$, in the left and right panels, respectively, compared to the measured values for 15,20 , and $50 \mathrm{~K}$. We also included the $\rho_{1 / 2, x x}$ and $\rho_{1 / 2, x y}$ contributions of the Ge:B epilayer and the substrate in the plot. There is good agreement between
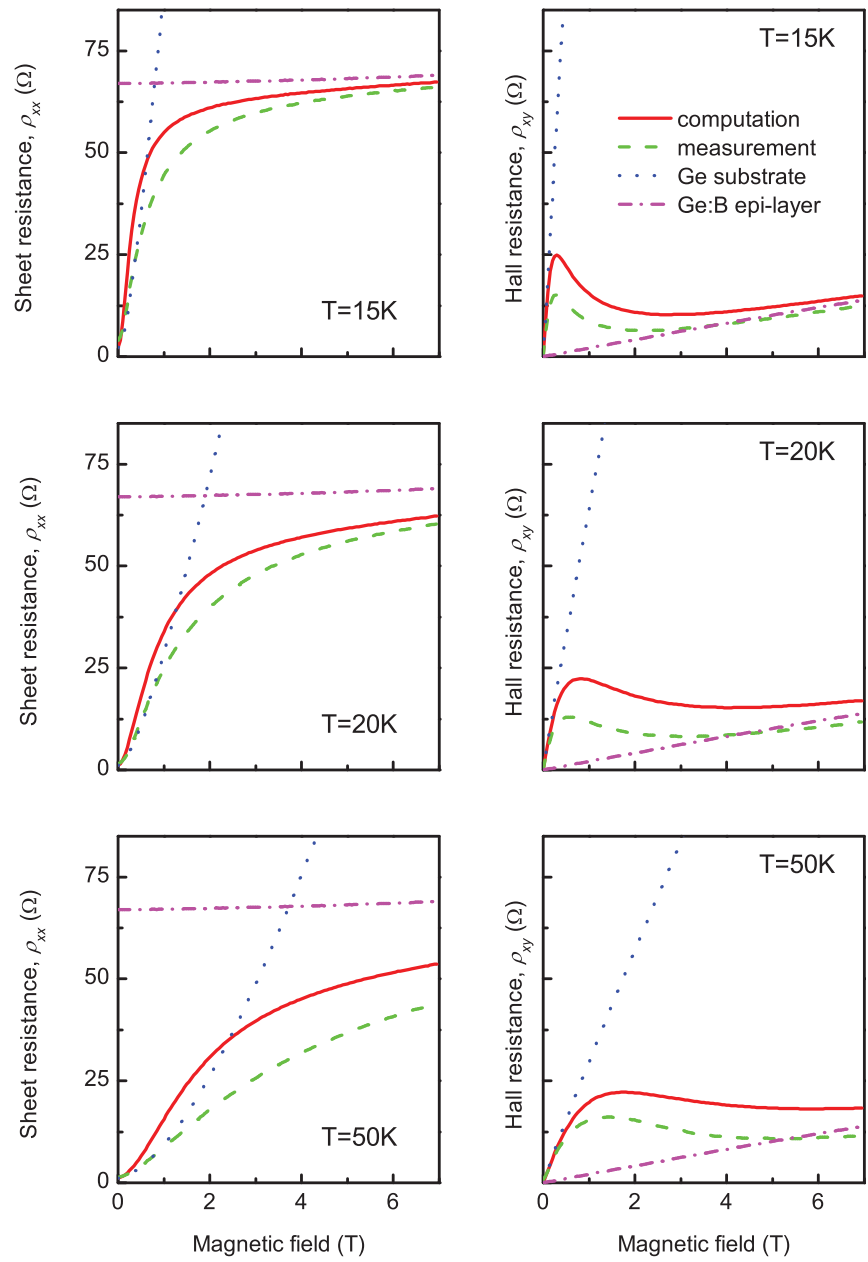

FIG. 6. (Color online) Sheet (left) and Hall resistance (right) versus magnetic field for different temperatures of the Ge:B sample affected by parallel conduction. Shown is the measurement, the computation according to Eqs. (2), and the individual contributions of the substrate and metallic epilayer.

the two-layer conduction model and the measurements of the Ge:B sample for both the $\rho_{x x}$ and $\rho_{x y}$ components. In particular, the low field domination of the Ge substrate layer as well as the saturation for the high field at the Ge:B epilayer contribution can be reproduced well by Eqs. (2). Thus, the model qualitatively demonstrates that parallel conduction through the substrate is also present in a sample with a degenerate, metallic, well-conducting epilayer in the extrinsic range of the underlying substrate. For the small quantitative differences between the computed and the actual experimental results, a major reason can be made out. The model Eqs. (2) strictly apply for a two-layer system, where both layers have the same in-plane geometry. Since the HB mesa, however, does not define such a geometry for the substrate conduction channel, differences between theory and experiment will occur.

From the results on the degenerate Ge:B reference sample we infer that in the case of metallic GeMn thin films deposited on the high-resistivity substrate, intrinsic properties of the GeMn epilayer may be directly derived in the freeze-out temperature regime of the substrate. For higher temperatures, however, care must be taken to separate the intrinsic properties 
of GeMn from the aforementioned effects arising due to the two-layer conduction.

\section{Influence of the sample geometry on MR measurements}

It was previously shown in Fig. 3(b) that the high-resistivity substrate of very pure Ge exhibits an extremely large MR effect up to $50000 \%$. However, reports of magnetotransport properties of high-purity Ge show that this large effect is not expected. ${ }^{22,23}$ To study the MR effect of the high-resistivity substrate further, we fabricated additional samples using a van der Pauw (vdP) geometry, as depicted in the schematic of Fig. 1(b). Interestingly, the MR effect of the vdP sample presented in Fig. 7 is now more than 20 times smaller than for the corresponding $\mathrm{HB}$ sample. Evidently, the large MR effect previously obtained in HB geometry is not an inherent physical property of the Ge substrate. The measurements taken in $\mathrm{vdP}$ geometry agree much better with the above-mentioned magnetotransport studies of Ge. ${ }^{22,23}$

Up to now we can only speculate about the reasons inducing the large MR effect observed in Fig. 3 for the HB samples. Most probably it is related to a redistribution of the current lines upon applying a magnetic field, similar to the effect observed in Ref. 17. Referring to the sample schematic depicted in Fig. 1(a), the relatively large, metallized voltage probes 2, $3,4,6,7$, and 8 have to be considered as effective short circuits within the sample volume, if conduction through the substrate is present. In a magnetic field these short circuits may be diminished as the electric field, and therefore the current flow, will get tangent to these areas. The result is an artificial increase of the sample resistance. Additionally the small length-to-width ratio of $l / w \approx 2$, basically given by the separation of contacts 1 and 5 and the width of contact 1 , may also favor a considerable Hall-effect-induced contribution to the intrinsic MR. ${ }^{24,25}$ Therefore the large MR effect of up to $50000 \%$, and with it also the anomalous shape of the Hall angle, have to be regarded as a purely extrinsic effect, which strongly relies on the specific HB geometry and the extent of parallel conduction through the substrate. It should be noted that attempts to fit these measurements by a two-carrier-type model, using reasonable parameters for the mobilites and concentrations of the two-carrier types were not successful. ${ }^{26}$

We also reinvestigated the GeMn thin-film sample in vdP geometry. Upon comparing it with the Ge substrate in Fig. 7 we now remark a pronounced difference between the MR
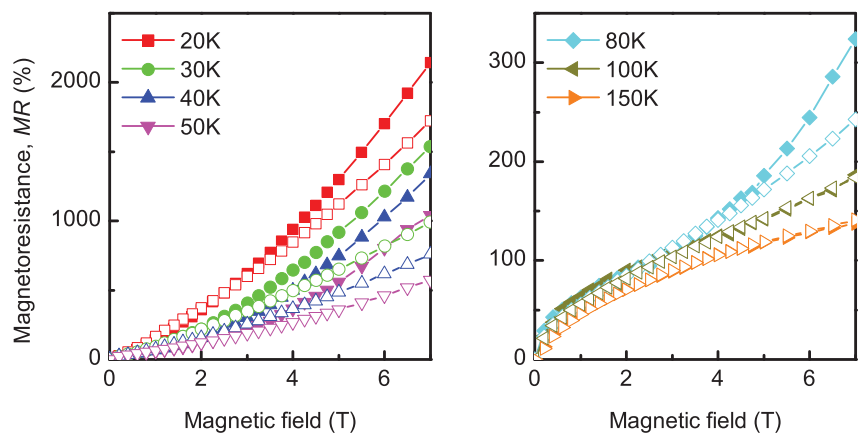

FIG. 7. (Color online) MR for various temperatures for the GeMn sample (open symbols) and the bare Ge substrate (filled symbols). measurement above approximately $3 \mathrm{~T}$ for temperatures up to $80 \mathrm{~K}$. The MR of the GeMn sample follows a more linear behavior, while the MR of the substrate still increases superlinearly above this field. At $100 \mathrm{~K}$ and higher temperatures both samples exhibit the same MR effect. We infer from this behavior that at least below $100 \mathrm{~K}$ transport properties of the GeMn epilayer become visible in the measurement. This may be due to the fact that in vdP geometry the volume of the epilayer is not restricted to the in-plane dimensions of the etched HB mesa, but extends over the whole chip area. Therefore the effective volume ratio of the epilayer to the substrate, hence the conductance ratio, is increased in the vdP geometry as compared to the HB geometry.

We thus conclude that a vdP geometry is to be favored over a HB mesa in the GeMn material system with its high probability of parallel conduction through the substrate. By using a vdP geometry the comparison of experimental data with an elaborate, $a b$ initio two-layer conduction model, extending the scheme outlined in Sec. III B, may enable a derivation of inherent transport properties of GeMn thin films, in spite of the dominant contribution of the Ge substrate.

\section{GeMn on $\boldsymbol{n}$-type Ge substrates}

The derivation of the GeMn transport properties would be much easier if the substrate contribution could be further reduced. To this end we fabricated another GeMn sample, which employs a Ge substrate with RT resistivity of $0.13 \Omega \mathrm{cm}$ having a well-defined concentration of Sb donors. The buildup of a rectifying interface between the $p$-type GeMn epilayer and the $n$-type substrate would isolate the epilayer electrically from the substrate. The 80-nm-thick GeMn epilayer has a Mn concentration of $10 \%$. To test the benefit of this concept, we again deposited a metallic Ge:B epilayer on such a substrate. Transport measurements of these samples were made in vdP geometry.

Figure 8 displays the temperature-dependent resistance of the second Ge:B sample together with the employed substrate. The curve shape is now in agreement with the metallic character of the Ge:B thin film over the entire temperature range and is clearly different from the substrate behavior. Note that the substrate resistance is actually a factor of 10 smaller than the resistance of the epilayer for temperatures above $30 \mathrm{~K}$, demonstrating the effectivity of the rectifying $p n$ barrier. The absence of parallel conduction through the substrate is also reflected in Hall measurements (not shown), which in contrast to the measurements depicted in Fig. 5 exhibit a linear Hall effect up to RT corresponding to the nominal doping concentration of the thin film.

Also shown in Fig. 8 is the resistance curve of the GeMn sample grown on the $n$-type substrate. Reliable measurement data are only available above $150 \mathrm{~K}$, since the electrical contacts become non-ohmic below this temperature. Nevertheless, in the available temperature range we note a clear difference of the resistance of this sample as compared to that of the substrate. The resistance quickly becomes larger, suggesting the absence of parallel conduction through the substrate. Magnetotransport studies of this sample reveal $p$-type conduction pointing toward the acceptor role of $\mathrm{Mn}$ in Ge. Hole concentrations ranging from $5 \times 10^{18} \mathrm{~cm}^{-3}$ to 


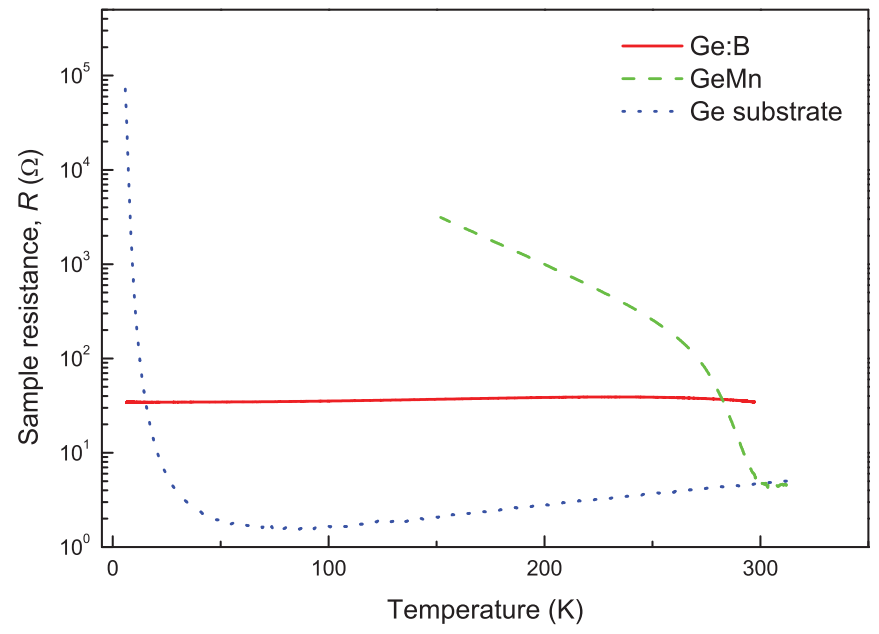

FIG. 8. (Color online) Sample resistance versus temperature for the GeMn sample (green dashed) and the Ge:B sample (red solid) grown on the n-type Ge substrate (blue dotted). The GeMn sample is affected by parallel conduction above $250 \mathrm{~K}$ as the $p n$ barrier becomes inefficient. The Ge:B sample shows metallic conduction up to RT.

$1.5 \times 10^{19} \mathrm{~cm}^{-3}$ for temperatures between 150 and $210 \mathrm{~K}$ could be deduced from the high field slope of Hall measurements (not shown). We could not identify any signs of a magnetization-induced AHE which, in general, pointing toward a low polarization of the holes, could also be related to the decreasing magnetic response of these types of GeMn thin films at accessible temperatures above $150 \mathrm{~K}$. This would be in line with the fact that this sample only exhibits unblocked superparamagnetism of only locally ferromagnetic, Mn-rich clusters with individual Curie temperatures of about RT. ${ }^{1,3}$

Figure 9 depicts the MR of the GeMn sample for three different temperatures. Interestingly, the MR changes only slightly with temperature and does not exceed $3 \%$ for the highest field, in contrast to a value of $120 \%$ for the sample affected by parallel conduction through the substrate, as depicted in Fig. 7. While this underlines the immense influence

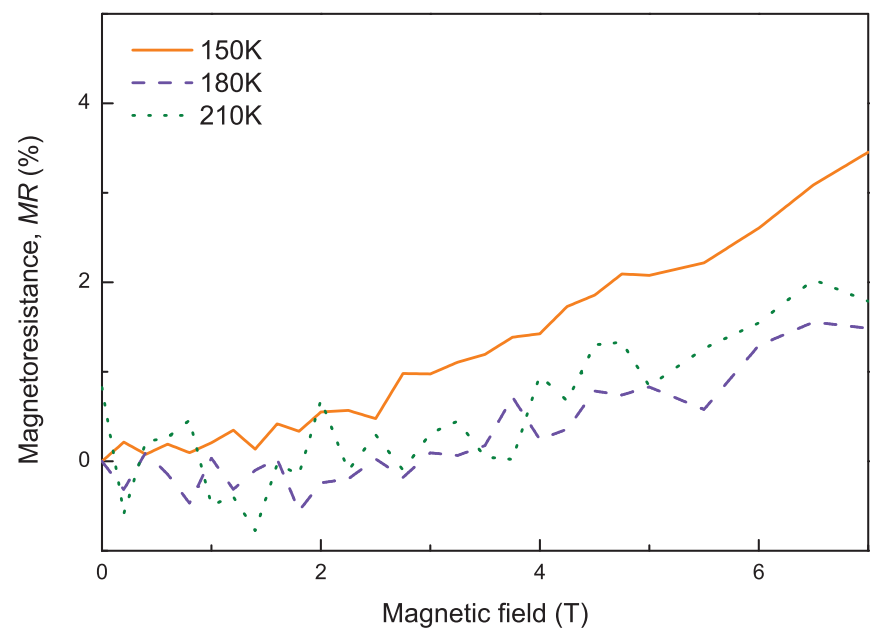

FIG. 9. (Color online) MR for various temperatures for the GeMn sample grown on the $n$-type Ge substrate. of the substrate contribution, it also demonstrates that the MR in our GeMn samples is apparently rather small. Its order of magnitude as well as the parabolic curvature could very well originate from the ubiquitous, normal orbital MR in a low mobility conductor.

The usage of an $n$-type substrate seems to unveil the intrinsic transport characteristics of our GeMn thin-film sample. Parallel conduction through the substrate is greatly reduced, compared to thin films fabricated on the high-resistivity Ge substrate. For degenerate epitaxial GeMn thin films a slightly $n$-type doped Ge substrate seems to be most adequate for transport studies. However, there are several drawbacks from a general, straightforward application of this approach to nondegenerate samples: The $p n$-barrier concept fails for higher temperatures, as indicated by the large drop of the resistance curve of the GeMn sample in Fig. 8 above $250 \mathrm{~K}$. A RT characterization of such devices is impossible, as parallel conduction through the substrate will be present. ${ }^{27}$ Furthermore, the formation of a space-charge region at the rectifying $p n$ interface leads to a depletion of charge carriers in a certain volume of the epilayer, depending on the carrier concentrations in the $p$ - and $n$-type regions. In the present case conduction in the epilayer is not affected, because of the large hole concentration, whereas for lower carrier concentrations or rather thin epilayers, conduction in the epilayer may be quenched. Moreover, a rapid thermal annealing process to obtain ohmic contacts could not be used for these types of samples, as this resulted in a direct contact to the substrate which short circuits the $p n$ barrier. A laser-assisted ultrashorttime annealing employed instead, however, did not provide ohmic contacts that work down to cryogenic temperatures. More sophisticated approaches might be explored to overcome this limitation and to also enable low-temperature measurements.

\section{SUMMARY}

In summary, the work presented here has shown that transport phenomena of nondegenerate GeMn thin films with hole concentrations around $10^{19} \mathrm{~cm}^{-3}$ fabricated on high-resistivity Ge substrates are not a consequence of the alloying of $\mathrm{Mn}$ with Ge. Instead it was found that the transport studies are severely influenced by parallel conduction through the substrate. This is in essence due to comparable resistances of the individual conducting layers. In this context, findings of extremely large magnetoresistance effects up to $50000 \%$ are related to an unfavorable measurement geometry. Measurements of a degenerate, $p$-type $\mathrm{Ge}: \mathrm{B}$ reference sample showed that despite the well-conducting epilayer, parallel conduction through the substrate is also present, significantly altering Hall and MR measurements which could only be understood in a two-layer conduction model. Parallel conduction through the substrate has been fully suppressed for the degenerate, $p$-type reference and partly for the GeMn thin-film sample, by using Sb-doped $n$-type substrates.

Our results hint toward the importance of a thorough characterization of the substrate properties in transport studies of GeMn thin films that are fabricated on Ge substrates. An 
increasing awareness and proper understanding of this problem may help to rule out possible misinterpretations. Such misinterpretations may a priori be avoided by using semi-insulating GaAs substrates with a resistivity larger than $10^{3} \Omega \mathrm{cm}$, delivering a small lattice mismatch to the Ge diamond lattice structure. A few reports on magnetotransport of various types of GeMn thin films employing GaAs substrates exist, $5,28-30$ however, out-diffusion of As or Ga is a critical issue. ${ }^{31,32}$ It was recently shown that this type of unintentional codoping may actually change the structural and also the magnetic properties of GeMn thin films. ${ }^{33}$

\section{ACKNOWLEDGMENTS}

The authors would like to thank the Deutsche Forschungsgemeinschaft for financial support via SPP 1285.
${ }^{1}$ D. Bougeard, S. Ahlers, A. Trampert, N. Sircar, and G. Abstreiter, Phys. Rev. Lett. 97, 237202 (2006).

${ }^{2}$ M. Jamet, A. Barski, T. Devillers, V. Poydenot, R. Dujardin, P. Bayle-Guillemaud, J. Rothman, E. Bellet-Amalric, A. Marty, J. Cibert, R. Mattana, and S. Tatarenko, Nat. Mater. 5, 653 (2006).

${ }^{3}$ D. Bougeard, N. Sircar, S. Ahlers, V. Lang, G. Abstreiter, A. Trampert, J. M. LeBeau, S. Stemmer, D. W. Saxey, and A. Cerezo, Nano Lett. 9, 3743 (2009).

${ }^{4}$ A. P. Li, C. Zeng, K. van Benthem, M. F. Chisholm, J. Shen, S. V. S. Nageswara Rao, S. K. Dixit, L. C. Feldman, A. G. Petukhov, M. Foygel, and H. H. Weitering, Phys. Rev. B 75, 201201 (2007).

${ }^{5}$ Y. D. Park, A. T. Hanbicki, S. C. Erwin, C. S. Hellberg, J. M. Sullivan, J. E. Mattson, T. F. Ambrose, A. Wilson, G. Spanos, and B. T. Jonker, Science 295, 651 (2002).

${ }^{6}$ A. P. Li, J. F. Wendelken, J. Shen, L. C. Feldman, J. R. Thompson, and H. H. Weitering, Phys. Rev. B 72, 195205 (2005).

${ }^{7}$ S. Zhou, D. Bürger, A. Mücklich, C. Baumgart, W. Skorupa, C. Timm, P. Oesterlin, M. Helm, and H. Schmidt, Phys. Rev. B 81, 165204 (2010).

${ }^{8}$ J. X. Deng, Y. F. Tian, S. M. He, H. L. Bai, T. S. Xu, S. S. Yan, Y. Y. Dai, Y. X. Chen, G. L. Liu, and L. M. Mei, Appl. Phys. Lett. 95, 062513 (2009).

${ }^{9}$ S. Zhou, D. Bürger, M. Helm, and H. Schmidt, Appl. Phys. Lett. 95, 172103 (2009).

${ }^{10}$ F. J. Morin and J. P. Maita, Phys. Rev. 94, 1525 (1954).

${ }^{11}$ M. B. Prince, Phys. Rev. 92, 681 (1953).

${ }^{12}$ O. Riss, A. Gerber, I. Y. Korenblit, A. Suslov, M. Passacantando, and L. Ottaviano, Phys. Rev. B 79, 241202 (2009).

${ }^{13}$ H. Fritzsche and K. Lark-Horovitz, Phys. Rev. 113, 999 (1959).

${ }^{14}$ The temperature dependence of the mobility has been neglected. Due to autocompensation we fitted the data with an $\exp \frac{E_{A}}{k_{B} T}$ law; see for example, J. S. Blakemore, Semiconductor Statistics, edited by H. K. Henisch (Pergamon, New York, 1962).

${ }^{15}$ H. H. Woodbury and W. W. Tyler, Phys. Rev. 100, 659 (1955).
${ }^{16}$ C. M. Hurd, The Hall Effect in Metals and Alloys (Plenum, New York, 1972).

${ }^{17}$ S. A. Solin, T. Thio, D. R. Hines, and J. J. Heremans, Science 289, 1530 (2000).

${ }^{18}$ M. M. Parish and P. B. Littlewood, Phys. Rev. B 72, 094417 (2005).

${ }^{19}$ M. M. Parish and P. B. Littlewood, Nature (London) 426, 162 (2003).

${ }^{20}$ J. Hu, M. M. Parish, and T. F. Rosenbaum, Phys. Rev. B 75, 214203 (2007).

${ }^{21}$ O. A. Golikova, B. Y. Moizhes, and L. S. Stil'bans, Sov. Phys. Solid State 3, 2259 (1962).

${ }^{22}$ C. Goldberg and R. E. Davis, Phys. Rev. 102, 1254 (1956).

${ }^{23}$ J. W. Gallagher and W. F. Love, Phys. Rev. 161, 793 (1967).

${ }^{24}$ R. F. Wick, J. Appl. Phys. 25, 741 (1954).

${ }^{25}$ H. H. Jensen and H. Smith, J. Phys. C 5, 2867 (1972).

${ }^{26}$ In the case of a two-carrier-type model Eqs. (2) apply when the sheet terms are replaced by their respective bulk counterparts, using the well-known standard expressions for $\rho_{1 / 2, x x}$ and $\rho_{1 / 2, x y}$.

${ }^{27}$ N. Pinto, L. Morresi, M. Ficcadenti, R. Murri, F. D’Orazio, F. Lucari, L. Boarino, and G. Amato, Phys. Rev. B 72, 165203 (2005).

${ }^{28}$ H. Li, Y. Wu, Z. Guo, P. Luo, and S. Wang, J. Appl. Phys. 100, 103908 (2006).

${ }^{29}$ C. Zeng, Y. Yao, Q. Niu, and H. H. Weitering, Phys. Rev. Lett. 96, 037204 (2006).

${ }^{30}$ C. Zeng, Z. Zhang, K. van Benthem, M. F. Chisholm, and H. H. Weitering, Phys. Rev. Lett. 100, 066101 (2008).

${ }^{31}$ R. Tsuchida, J. T. Asubar, Y. Jinbo, and N. Uchitomi, J. Cryst. Growth 311, 937 (2009).

${ }^{32}$ Y. D. Park, A. Wilson, A. T. Hanbicki, J. E. Mattson, T. Ambrose, G. Spanos, and B. T. Jonker, Appl. Phys. Lett. 78, 2739 (2001).

${ }^{33}$ I.-S. Yu, M. Jamet, T. Devillers, A. Barski, P. Bayle-Guillemaud, C. Beigné, J. Rothman, V. Baltz, and J. Cibert, Phys. Rev. B 82, 035308 (2010). 